\title{
Use of hollow fiber-optic elements in information input systems with optical coding
}

[Vituleva ES, Baipakbaeva ST, Suleimenov IE]

\begin{abstract}
An improved lightguide element design for a keyboard with optical coding is proposed in the article, based on the phenomenon of light reflection from the interface of two optical transparent media. A feature of this element is the use of relatively large values of the reflection coefficient from the medium interface at large angles of incidence, which is expressed, in particular, by the Fresnel formulas. This effect occurs also in the case when light passes from an optically less dense medium (air) to an optically denser (polymer), which, in particular, makes it possible to use a hollow polymer tube as a lightguide element. A differential equation is obtained that describes the shape of the initial section of an improved lightguiding element of a given type, as well as its analytical solution. This type of solution corresponds to a surface optical trap whose shape corresponds to multiple reflection of the rays from the same non-planar surface.
\end{abstract}

Keywords - media interface, reflection coefficient, lightguide elements, Fresnel formulas, optical trap

\section{Introduction}

In articles [1, 2], a new principle of a keyboard operation with optical coding was proposed, based on the use of reversibly deformable lightguiding elements. The number N1 of such elements in the keyboard layout is equal to the number of columns in the matrix which elements correspond to the keys. The light guide elements are located perpendicular to the segments of the photovoltaic panels, which number is equal to the number of rows in the indicated matrix, Fig. 1. Structurally, the lightguiding elements are located on top of the photovoltaic panel consisting of N2 segments, where N2 is the number of lines.

Suleimenov Ibragim

Almaty university of Power Engineering and Telecommunications Kazakhstan

Baipakbayeva Saltanat

Almaty university of Power Engineering and Telecommunications Kazakhstan

Vituleva Elizaveta

The Kazakh National Research Technical University after K.I.SatpaevKazakhstan

\section{Theory}

In articles [1, 2], a new principle of a keyboard operation with optical coding was proposed, based on the use of reversibly deformable lightguiding elements. The number N1 of such elements in the keyboard layout is equal to the number of columns in the matrix which elements correspond to the keys. The light guide elements are located perpendicular to the segments of the photovoltaic panels, which number is equal to the number of rows in the indicated matrix, Fig. 1. Structurally, the lightguiding elements are located on top of the photovoltaic panel consisting of N2 segments, where N2 is the number of lines.

The identification of the pressed key is made by alternately supplying short-term voltage pulses to the LEDs connected to the light-guiding elements; the duration of each pulses is equal to $\mathrm{T} / \mathrm{N} 1$, where $\mathrm{T}$ is the duration of the polling cycle of the columns. When the fiber section is deformed, located at its intersection with a particular panel segment, an alternating voltage is applied to it, i.e. the line is identified by the line number on which the pressed key is located, and the column number by the duration of the recorded sequence of pulses delay relative to the start of the cycle.

This approach [1,2] allows the product to be operated in two modes - the charging mode of the gadget, in which the photoelectric panel generates a constant voltage by converting the energy of solar radiation, and in the information input mode in which the output of each of the segments on which the pressed keys are located, a pulse-periodic signal is generated.

Earlier [2] it was experimentally shown that it is permissible to use hollow tubes made of a transparent polymeric material as a deformable fiber.

We emphasize that a hollow polymer tube is not a lightguide in the strict sense of this term, since the effect of total internal reflection is not used in such an element when rays are propagated. However, provided that the directivity pattern of the light source is rather narrow, the radiation losses during propagation over small distances are relatively small. This follows from the fact that when the rays fall onto the interface between media at a large incidence angle $\varphi$ (Fig. 2), the reflection coefficient is large enough even when the medium filling the light-conducting element of the type under consideration (air) is optically less dense than the environment from which the element itself is formed (polyethylene or polystyrene tube).

However, such a shape of the lightguiding element, in which the phenomenon radiation reflection from the interface of an optically less dense and denser medium is used, is not optimal. In this paper, a shape of the surface is obtained, which makes it possible to optimize the shape of a hollow fibroptic element made of a transparent polymer material. The 
basis for this is the solution of the differential equation describing the shape of one of the varieties of the optical trap.

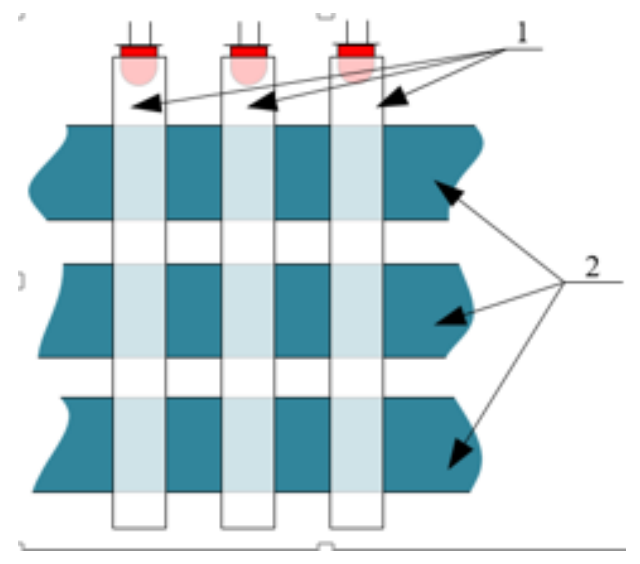

Figure 1. Diagram of the relative position of the lightguiding elements (1) and segments of the photovoltaic panel (2)

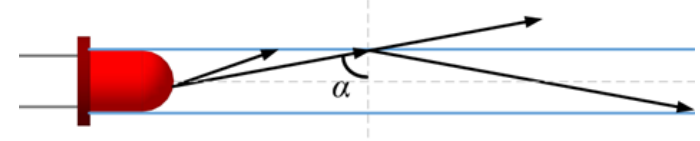

Figure 2. Scheme of rays propagation in a hollow lightguide element at large angles of incidence $(\alpha)$.First, confirm that you have the correct template for your paper size

\section{Results and discussions}

The mechanism of a hollow polymer tube operation as a light guide element is illustrated in Fig. 3, which shows the dependence of the reflection coefficients of RS, RP s- and ppolarized waves on the angle of incidence; It is assumed that light passes from an optically less dense medium $\mathrm{n} \cong 1$ to an optically denser medium. In the calculations it is assumed that the refractive index of the material used (polystyrene) is 1.59 . The working range of incidence angles is shown in the figure, in which the average values of the reflection coefficient exceed 0.5 .

When constructing Fig. 3 we used the Fresnel formulas [3] for s-and p-polarized waves

(1)

$$
R_{s}=\frac{\sin ^{2}(\alpha-\beta)}{\sin ^{2}(\alpha+\beta)}
$$

$$
R_{P}=\frac{\operatorname{tg}^{2}(\alpha-\beta)}{\operatorname{tg}^{2}(\alpha+\beta)}
$$

where $\alpha$ and $\beta$ are the angles of incidence and reflection, related to each other by Snell's law (Fig. 2)

$$
\frac{\sin \alpha}{\sin \beta}=\frac{n_{2}}{n_{1}}
$$

from
Accordingly, the condition formulated above is written as

$$
\varepsilon_{0}=\varepsilon_{1}-\varepsilon_{2}=\text { const }
$$

where $\mathrm{n} 1$ and $\mathrm{n} 2$ are the refractive indices of the media in which the incident and refracted waves develop, respectively, which gives a relationship

$$
\beta=\arcsin \left(\frac{\sin \alpha}{n}\right)
$$

where $\mathrm{n}$ is the relative refractive index of the above media.

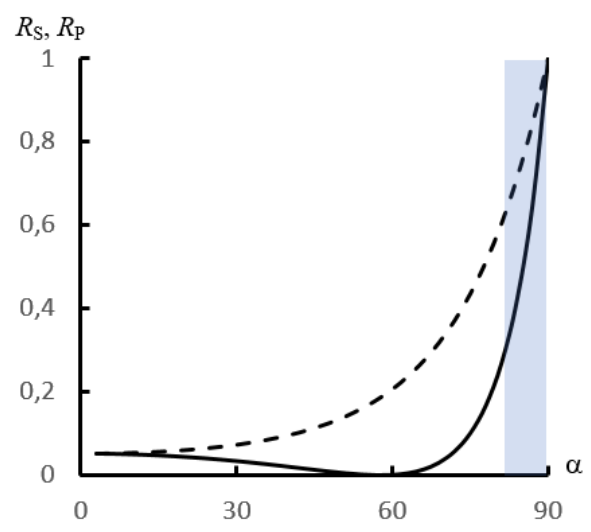

Figure 3. Dependences of the reflection coefficients s(dashed line) and p-polarized wave (solid line) on the media interface with refractive indices $n=1$ and $n=1.59$

We obtain a differential equation describing the profile of an optical trap of the simplest type. We will consider the case when all the rays lie in the Oxy plane, which allows us to solve the two-dimensional problem. The condition that allows us to obtain the required equation is the constancy of the angle of incidence on the interface between media of all rays emitted by a point source of light (it is assumed that it is located at the origin).

For all rays emitted by a point source located at the origin,

$$
\operatorname{tg} \varepsilon_{1}=\frac{f(x)}{x}
$$

where $f(x)$ is the function describing the required curve; $x$ is the coordinate, $\varepsilon_{-} 1$ is the angular variable.

The tangent of the slope $\varepsilon_{2}$ of the tangent to the desired curve at the point $x$ is

(6)

$$
\operatorname{tg} \varepsilon_{2}=\left.\frac{d f}{d x}\right|_{x}
$$

$$
\text { ) }
$$


or

$$
\operatorname{tg} \varepsilon_{0}=\frac{\frac{f(x)}{x}-\frac{d f}{d x}}{1+\frac{f(x) d f}{x d x}}
$$

(9)

We introduce the notation

Then the required differential equation is written as

$$
\frac{d f}{d x}=\frac{\frac{f(x)}{x}-g}{1+g \frac{f(x)}{x}}
$$

Equation (11) is homogeneous, so we use the substitution

$$
f=w x
$$

Whence

$$
x \frac{d w}{d x}+w=\frac{w-g}{1+g w}
$$
form

By simple transformations, expression (13) reduces to the

$$
\frac{1+g w}{1+w^{2}} d w=-g \frac{d x}{x}
$$

or

$$
\int \frac{1}{1+w^{2}} d w+g \int \frac{w}{1+w^{2}} d w=-g \int \frac{d x}{x}+C
$$

In the last formula, all the integrals are tabular; we have

$\operatorname{arctg}(w)+\frac{g}{2} \ln \left(1+w^{2}\right)=-g \ln x+C$

Whence

The dependences $f(x), \frac{f(x)}{x}, \frac{d f}{d x}$ on the longitudinal coordinate $\mathrm{x}$ for two particular cases are shown in Fig. 4a, b. The scale of the axis is chosen so that the geometric similarity with real spatial coordinates is preserved, i.e. the solid curves in Fig. 4 are geometrically similar to the generators of the lightguiding element.

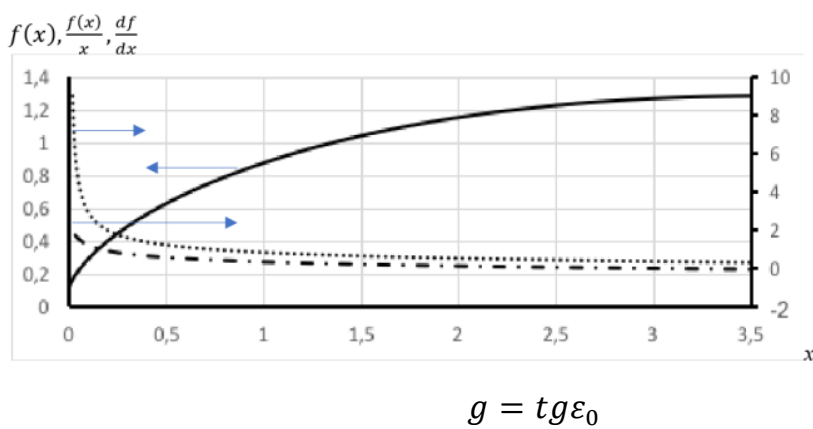

Figure $4 \mathrm{a}$. The profile of the solution of equation (11) is a solid line, the ratio $\frac{f(x)}{x}$ (dotted line) and the derivative $\frac{d f}{d x}$ (dot-dash line) at $\mathrm{A}=10, \mathrm{~g}=0.358$

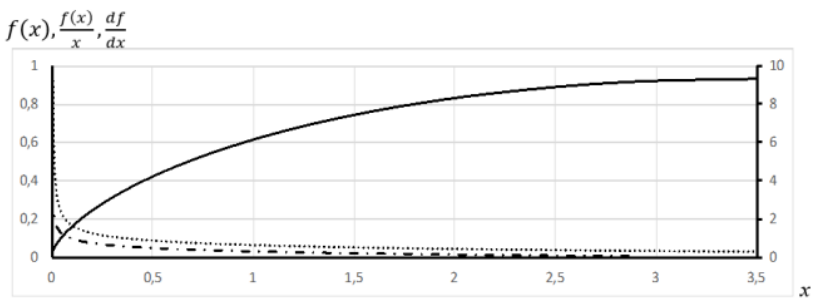

Figure $4 \mathrm{~b}$. The solution profile of equation (11) is a solid line, the ratio $\frac{f(x)}{x}$ (dotted line) and the derivative $\frac{d f}{d x}$ (dashed line) for $\mathrm{A}=10, \mathrm{~g}=0.258$

It is seen that as the value of $g$ decreases, that is, the angle of incidence of the rays emitted by the point source on the surface under consideration is reduced, the profile is pressed against the axis Ox. Accordingly, it is possible to select a sufficiently small value of the angle of incidence $\varepsilon_{-} 0$, which is common for all rays.

The limiting factor here is the real size of the source, which is not really a point source. More precisely, the limiting factor is the ratio of the linear dimensions of the light source (in relation to the case of using a lightguide element in keypads with optical coding, the LED) and the permissible linear dimensions of the lightguiding element proper. This is illustrated in Fig. 5, which shows the profiles for the same value of $g$ as in Fig. 4c, but for a different value of the constant A. It can be seen that these profiles are similar: as the coefficient $\mathrm{A}$ is changed, the dimensions of the figure along the two coordinate axis proportionally increase.

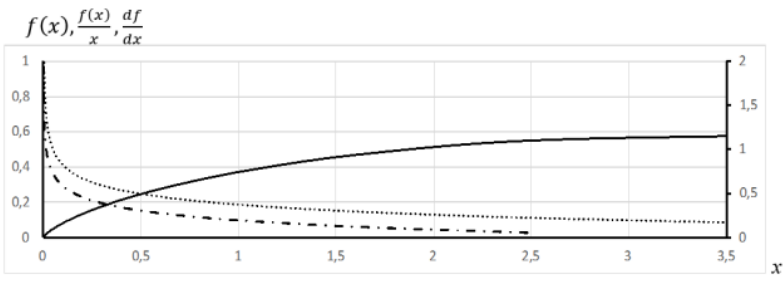

Figure 4c. The profile of the solution of equation (11) is a solid line, the ratio $\frac{f(x)}{x}$ (dashed line) and the derivative $\frac{d f}{d x}$ (dash-dot line) at $\mathrm{A}=10, \mathrm{~g}=0.158$ 


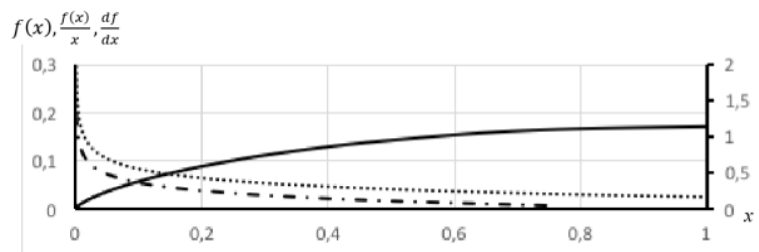

Figure 5. The profile of the solution of equation (11) is a solid line, the ratio $\frac{f(x)}{x}$ (dashed line) and the derivative $\frac{d f}{d x}$ (dash-dot line) for $\mathrm{A}=3, \mathrm{~g}=0.158$

\section{Conclusion}

Thus, the transition to sophisticated geometry really increases the efficiency of optical signal transmission in hollow lightguide elements made of transparent polymeric materials. This is achieved through the use of a lightguiding element, the inner and outer surface of which is a figure of rotation, the generatrix of which is the curve given by the solution of equation (11). A certain disadvantage of using this approach is the rather large transverse dimensions of the tube of a non-constant cross section. Partially to eliminate this disadvantage it can be used an element with an ellipsoidal cross section, since the size of the lightguide element in use can be the same width as the keyboard keys at a relatively small thickness.

The geometric form of such an element is given by the following relation (in cylindrical coordinates)

where $f(\rho)$ is the curve corresponding to the solution of equation (11); generates, and the function $R(\varphi)$ describes an ellipsoidal section.

An obvious advantage of this kind of elements is the possibility of their production from the cheapest material (polyethylene) according to well-developed technologies at the moment.

\section{References}

[1] Sh.B. Kabdushev, I.E. Suleimenov, D.B. Shaltykova, I.V. Iglikov, B.B. Ermukhambetova, S.T. Baipakbaeva, G.A. Mun, A.A. Nurtazin, "System input information on the basis of solar photovoltaic panels," WSEC -2017, vol. 3, pp. 201-206, June 2017. (references)

[2] S.T. Baipakbaeva, "Possible-strategy of small green energy concept in the Republic of Kazakhstan," IASS Ann. Int. Acad. Conf. on Silk Road, vol. 1, pp. 257-268, November 2017. (references)

[3] D.V.Sivukhin, General Course of Physics: Optics, M: Nauka, 1980.

About Author (s):

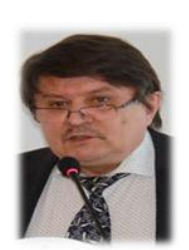

Professor I.E. Suleimenov Candidate of Physical and Mathematical Sciences

Doctor of Chemistry sciences, Academician of National Engineering Academy of the Republic of Kazakhstan

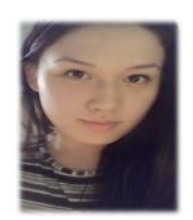

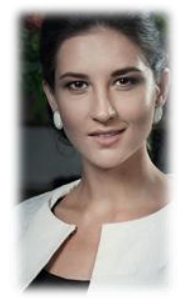

E. Vituleva senior lecturer of The Kazakh National Research Technical University after K.I.Satpaev.

$$
z=f(\rho) R(\varphi)
$$

